\section{VARIAÇÃO ANATÔMICA DO TRONCO CELÍACO E DO SISTEMA ARTERIAL HEPÁTICO: UMA ANÁLISE POR EXAMES DE IMAGEM}

\section{ANATOMICAL VARIATIONS OF THE CELIAC TRUNK AND THE HEPATIC ARTERIAL SYSTEM: AN IMAGING ANALYSIS}

\author{
Victor Antonio Kuiava1, Senair Alberto Ambros ${ }^{1,2}$ \\ Mateus Picada Corrêa ${ }^{1,3}$, Daniel Navarini ${ }^{1,4}$
}

\section{RESUMO}

Introdução: As variações anatômicas das artérias hepáticas e do tronco celíaco são de grande importância para cirurgias laparoscópicas, transplantes hepáticos, intervenções radiológicas e tratamento de lesões abdominais. O grande aumento de intervenções minimamente invasivas deixou os atos cirúrgicos com menos espaço para o reconhecimento de estruturas anatômicas.

Métodos: Foi realizado um estudo retrospectivo do banco de dados do Hospital São Vicente de Paulo durante o ano de 2016, analisando imagens abdominais de tomografia computadorizada com contraste e angiotomografias que envolvem a aorta abdominal e seus ramos - um total de 461 imagens foram analisadas.

Resultados: Dos 461 pacientes analisados, 86,9\% apresentaram a conformação usual do tronco celíaco - cuja anatômica é a origem tríplice com as artérias gástrica esquerda, esplênica e hepática comum e artéria mesentérica superior se originando sozinha da aorta abdominal. Dentre as anatomias anômalas (13\%), o padrão mais comum desses ramos foi a presença em $4,5 \%$ de um tronco hepatomesentérico e um tronco gastroesplênico. No sistema arterial hepático a conformação mais prevalente foram as artérias hepáticas direita e esquerda sendo ramos da hepática próprias em $66,3 \%$. Das alterações anatômicas $(33,2 \%)$, as mais comuns foram a presença de uma artéria hepática esquerda acessória ramo da artéria gástrica esquerda $(7,8 \%)$.

Conclusão: Variações anatômicas do sistema arterial hepático e do tronco celíaco são prevalentes, podendo apresentar diversos arranjos organizacionais.

Palavras-chave: Artéria celíaca; artéria mesentérica superior; artéria hepática; variação anatômica

\section{ABSTRACT}

Introduction: Anatomical variations in the hepatic arteries and the celiac trunk are of great importance for laparoscopic surgeries, liver transplants, radiological interventions and treatment of abdominal injuries. A large increase in the number of minimally invasive interventions hampered the recognition of anatomical structures in surgical procedures.

Methods: A retrospective study was performed using the 2016 São Vicente de Paulo Hospital database of contrast-enhanced abdominal computed tomography images and computed tomography angiographies showing the abdominal aorta and its branches. In total, 461 images were analyzed.

Results: Of the 461 patients analyzed, $86.9 \%$ had usual conformation of the celiac trunk, which trifurcates into the left gastric artery, the common hepatic artery and the
Clin Biomed Res. 2019;39(3):225-229

1 Faculdade de Medicina, Universidade de Passo Fundo (UPF). Passo Fundo, RS, Brasil.

2 Departamento de Radiologia, Hospital São Vicente de Paulo (HSVP).

Passo Fundo, RS, Brasil.

3 Instituto de Cirurgia Vascular (Invasc). Passo Fundo, RS, Brasil.

4 Clínica Gastrobese. Passo Fundo. RS, Brasil.

Autor correspondente: Victor Antonio Kuiava victorkuiava@gmail.com Faculdade de Medicina, Universidade de Passo Fundo (UPF)

Rua Teixeira Soares, 817. 99010-080, Passo Fundo, RS, Brasil. 
splenic artery, while the superior mesenteric artery originates alone from the abdominal aorta. Among the cases of anomalous anatomy (13\%), the most common pattern in these branches was the presence of a hepatomesenteric trunk and a gastrosplenic trunk in $4.5 \%$. In the hepatic arterial system, the most prevalent conformation was the right and left hepatic arteries being branches of the hepatic artery proper in $66.3 \%$. Of all anatomical variations (33.2\%), the most common were the presence of a left accessory hepatic artery of the left gastric artery (7.8\%).

Conclusion: Anatomical variations in the hepatic arterial system and the celiac trunk are common, having different structural arrangements.

Keywords: Celiac artery; mesenteric artery, superior; hepatic artery; anatomical variation

Os grandes avanços em técnicas cirúrgicas como a cirurgia por vídeo e a robótica, transplantes de órgãos e intervenções radiológicas abriram portas para importantes conquistas médicas. As variações anatômicas das artérias hepáticas e do tronco celíaco são de grande importância para cirurgias laparoscópicas, transplantes hepáticos, intervenções radiológicas e tratamento de lesões abdominais. Essas variações, na ordem de 15 bem descritas na literatura, seriam decorrentes de alterações do padrão mais comum da embriogênese durante vários processos de formação dos humanos, geralmente associados a pouca ou nenhuma repercussão clínica ${ }^{1-3}$

O grande aumento de intervenções minimamente invasivas deixaram os atos cirúrgicos com menos espaço para o reconhecimento de estruturas anatômicas. Portanto, há um aumento da necessidade do conhecimento variações anatômicas para se diminuir o risco de lesões iatrogênicas. Informações como diâmetro e comprimento de vasos tornam-se importantes, ainda mais quando estruturas como o tronco celíaco e artéria mesentérica superior situam-se muito próximas, com média de $1,2 \mathrm{~cm}$ de distância ${ }^{4-6}$.

Os órgãos do trato intestinal, na porção abdominal, têm como vascularização três artérias principais, o tronco celíaco e as artérias mesentéricas superiores e inferior ${ }^{7,8}$. Sendo o tronco celíaco o mais superior dessas três artérias, se origina na altura de T12 a L1 e os três ramos mais comuns originados por essa artéria são a artéria gástrica esquerda, artéria hepática comum e artéria esplênica. Essa conformação é vista em torno de $89 \%$ dos casos da literatura, uma bifurcação é vista em $11 \%$ e a ausência do tronco em $0,2 \%{ }^{1,8,9}$.

Já a conformação do sistema arterial hepático é composta principalmente por uma artéria hepática esquerda e uma direita, que são ramos da artéria hepática própria, originada após a bifurcação da artéria gastroduodenal da hepática comum. A artéria hepática própria se bifurca no ligamento hepatoduodenal, se transformando em hepática direita e esquerda ${ }^{3,10}$.
O objetivo desse estudo é analisar a prevalência de variações anatômicas da ramificação do tronco celíaco e do sistema arterial hepático, além de avaliar outros dados, como o comprimento desses vasos, a distância entre eles e a esqueletopia dessas artérias. Os dados serão analisados a partir de imagens de pacientes que foram submetidos a exames de tomografia computadorizada com contraste oi angiotomografia.

\section{MÉTODOS}

O estudo foi realizado a partir do banco de dados do Hospital São Vicente de Paulo (HSVP), localizado na cidade de Passo Fundo, Rio Grande do Sul. Neste local foram selecionados exames por imagem de pacientes submetidos tomografia computadorizada com contraste de abdômen total ou de abdome superior e angiotomografia de abdome referente ao ano de 2016. O programa de computador que foi utilizado para a reconstrução e visualização das imagens foi através do programa de computador PACS Aurora - Arya.

No total de 511 reconstruções foram analisadas. Sendo 50 reconstruções excluídas por motivos de pacientes repetidos, imagens com qualidade inferior - principalmente devido a alterações no tempo de enchimento, e situações patológicas que possam a vir a alterar a anatomia da região, como gastrectomias, hepatectomias.

A análise dos padrões anatômicas foram realizadas por uma discussão interdisciplinar entre um radiologista, cirurgião vascular e um cirurgião digestivo. As variáveis analisadas foram o padrão de ramificação arterial do tronco celíaco e do sistema arterial hepático, comprimento do tronco celíaco e maior o diâmetro do tronco celíaco e da mesentérica superior. O projeto de pesquisa recebeu aprovação pelo Comitê de Ética em Pesquisa da Universidade de Passo Fundo.

\section{RESULTADOS}

Dos 461 pacientes analisados, $86,9 \%$ apresentaram a conformação usual do tronco celíaco - cuja anatomia é a origem tríplice com as artérias gástrica esquerda, esplênica e hepática comum e artéria 
mesentérica superior se originando sozinha da aorta abdominal. Dentre as anatomias anômalas (13\%), o padrão mais comum desses ramos foi a presença em $4,5 \%$ de um tronco hepatomesentérico e um tronco gastroesplênico, seguido da artéria gástrica esquerda se originado direto da aorta $(3 \%)$ e a fusão dos troncos celíaco e mesentérico em uma só estrutura vascular $(0,6 \%)$ - Tabela 1.

No sistema arterial hepático a conformação mais prevalente foi as artérias hepáticas direita e esquerda sendo ramos da hepática próprias em $66,3 \%$. Das alterações anatômicas (33,2\%), as mais comuns foram a presença de uma artéria hepática esquerda acessória ramo da artéria gástrica esquerda $(7,8 \%)$ e a artéria hepática esquerda ramo da gástrica esquerda $(5,8 \%)$, seguida pela artéria hepática direita ramo da mesentérica superior $(5,6 \%)$ (tabela 2).

A esqueletopia mais frequente do tronco celíaco e da mesentérica superior foi a vértebra $L 1$ em $42 \%$ e $66 \%$. A distância média entre as duas artérias foi de 7,84 mm (IC de 95\%, 7,69-7,98). O comprimento médio do tronco celíaco foi de $21,9 \mathrm{~mm}$ (IC de $95 \%$, 21,7-22,2), associado a um diâmetro médio de $8,8 \mathrm{~mm}$ (IC de 95\%, 8,68-8,9). A artéria mesentérica superior teve um diâmetro médio de $9,86 \mathrm{~mm}$ (IC de $95 \%$, 9,7-10,03) - tabela 3.

No presente estudo foram encontradas 16 padrões artérias hepáticos e do tronco celíaco não descritos na literatura - como a hepática comum sendo ramo da mesentérica superior e a hepática esquerda ramo da gástrica esquerda.

Tabela 1: Distribuição dos padrões artérias mais prevalentes do tronco celíaco.

\begin{tabular}{lcc}
\hline Padrão arterial & Número de casos & Porcentagem \\
\hline Anatomia normal (Tronco Tríplice + MS); & 401 & 86.98 \\
Tronco hepatoesplênico + GE + MS; & 14 & 3.04 \\
Tronco hepatomesentérico + Tronco gastroesplênico & 21 & 4.56 \\
Hepática comum + Tronco gastroesplênico + MS; & 6 & 1.30 \\
Outras variações & 19 & 4.12 \\
\hline
\end{tabular}

Tabela 2: Distribuição dos padrões artérias mais prevalentes do sistema arterial hepático.

\begin{tabular}{lcc}
\hline Padrão Arterial & Número de casos & Porcentagem \\
\hline Anatomia normal & 306 & 66.38 \\
AHE acessória ramo da AGE; & 36 & 7.81 \\
AHE ramo da AGE; & 27 & 5.86 \\
AHD ramo da AMS; & 26 & 5.64 \\
AHC ramo da AMS; & 21 & 4.56 \\
AHD Acessória ramo da AMS; & 11 & 2.39 \\
AHD ramo da AMS e AHE Acessória ramo da AGE; & 8 & 1.74 \\
AHD ramo da AMS e AHE ramo da AGE; & 5 & 1.08 \\
Outras variações & 21 & 4.56
\end{tabular}

AHE - Artéria Hepática Esquerda; AHD - Artéria Hepática Direita; AHC - Artéria Hepática Comum; AGE - Artéria Gástrica Esquerda; AMS Artéria Mesentérica Superior.

Tabela 3: Distribuição de maior diâmetro, comprimento, esqueletopia e distância entre as emergências do tronco celíaco e da artéria mesentérica superior.

\begin{tabular}{lcc}
\hline & Tronco Celíaco & Artéria Mesentérica Superior \\
\hline Diâmetro & $8,8 \mathrm{~mm}(\mathrm{IC}$ de $95 \%, 8,68-8,9)$ & $9,86 \mathrm{~mm}(\mathrm{IC}$ de 95\%, 9,7-10,03) \\
Comprimento & $21,9 \mathrm{~mm}$ (IC de 95\%, 21,7-22,2) & - \\
Esqueletopia & $\mathrm{L} 1$ & $\mathrm{~L} 1$ \\
Distância & $7,84 \mathrm{~mm}(\mathrm{IC}$ de $95 \%, 7,69-7,98)$ & \\
\hline
\end{tabular}




\section{DISCUSSÃO}

A anatomia usual do tronco celíaco e do sistema arterial hepático consiste na presença de três artérias oriundas do tronco celíaco, que são a artéria gástrica esquerda, a artéria esplênica e a artéria hepática comum $^{3,7}$. Por sua vez, a artéria hepática comum se bifurca em duas, uma hepática própria - que dará origem às artérias hepáticas direita e esquerda e uma artéria gastroduodenal.

Alterações vasculares nas artérias hepáticas são encontradas em $21 \%$ dos pacientes. São alterações na circulação hepática, podendo ser artérias acessórias ou substitutivas. Os padrões mais conhecidos são a presença de uma artéria hepática direita originada do mesentérico superior e / ou ramo hepático esquerdo da artéria gástrica esquerda ${ }^{4-6,8}$.

No maior estudo das variações do tronco celíaco e do sistema arterial hepático, realizado com 5002 pacientes na Coréia, há a descrição de 15 padrões artérias consolidados para esse sistema de circulação. Contudo, devido a diferenças étnicas e regionais desse estudo com a realidade brasileira, ele apresenta limitações - a prevalência dos padrões artérias encontrados no presente estudo são diferentes dos coreanos, sendo que a presença de um tronco gastroesplênico e um hepato mesentérico é maior que a obtida nesse estudo ${ }^{1}$. Além disso, há padrões arteriais que nem foram descritos pela literatura, sendo que elas variações anatômicas podem ser situações delicadas para cirurgias que envolvam o abdome superior, sendo que podem levar a situações complexas dependendo do procedimento e da gravidade da intervenção $0^{11-13}$.

Variações arteriais são devidas a alterações do padrão habitual da embriogênese, levando a formações vasculares peculiares, como artérias acessórias ou substitutivas. Geralmente, essas alterações são assintomáticas, não causando repercussão clínica significativa, porém, em situações especiais ou no manejo cirúrgico, podem induzir iatrogenia ${ }^{7}$.

As principais complicações estão relacionadas ao não reconhecimento de ramos arteriais anormais durante procedimentos de cirurgia hepática, são a não correta localização do ramo da artéria hepática direita da artéria mesentérica superior, cuja localização é retroportal. Essa conformação anatômica pode induzir a iatrogenia durante a cirurgia, como clampeamento desnecessário ou perfurações ${ }^{6-8,14,15}$.
A viabilidade de um transplante de fígado está intimamente relacionada ao sucesso das anastomoses vasculares realizadas. As complicações vasculares raramente ocorrem, contudo são o principal diagnóstico etiológico para insuficiência de órgãos fulminantes, fístulas e bacteremia sem causa etiológica devido ao comprometimento do suporte sanguíneo tanto a entrada quanto a saída. A artéria hepática é o sítio arterial mais comum de complicações vasculares no transplante. Suas complicações mais comuns são a trombose, que ocorre em $15,8 \%$ dos casos e a estenose, em 7,8\%. Quando presentes, estão associados à perda de órgão em $53 \%$ e mortalidade em $33-50 \%$ dos pacientes ${ }^{12,16,17}$.

Os exames de imagem pré-operatórios no transplante hepático são fundamentais para a análise do sistema vascular, tanto arterial quanto venoso. A identificação correta das variações anatômicas permite a redução de situações cirúrgicas inesperadas pelo cirurgião, às vezes difíceis de serem manejadas ou impossíveis de transplantar ${ }^{16,18,19}$. Esta avaliação é geralmente realizada por angiotomografia com análise arterial, portal e tardia ou por angiografia celíaca e mesentérica para as artérias hepáticas e nas veias portais ou por ressonância magnética para as artérias venosas e portais. e ducto biliar ${ }^{11,14,15}$.

O manejo cirúrgico dessas situações de variações anatômicas no transplante de fígado começa primeiramente com a identificação préoperatória desses padrões através de exames de imagem. No ato cirúrgico, essas variações são tratadas com ressecção do órgão com grande margem de tecido hepático com extensões do ligamento hepatoduodenal, tronco celíaco, artérias hepáticas acessórias e artéria hepática direita e esquerda. Remoções maiores de tecido hepático e tecidos peri-hepáticos diminuem a possibilidade de lesões vasculares, e reduzem complicações como trombose e estenose da artéria hepática ${ }^{7,10}$.

A variações anatômicas do sistema arterial hepático e do tronco celíaco são situações prevalentes e geralmente são condições assintomáticas. Contudo, a não correta identificação de desvios anatômicos pode levar a lesões iatrogênicas durante procedimentos intervencionistas. Associado a isso o conhecimento de diversos padrões artérias é fundamental para diminuir a morbidade e a mortalidade durante grandes atos cirúrgicos como o transplante hepático. 


\section{REFERÊNCIAS}

1. Song S-Y, Chung JW, Yin YH, Jae HJ, $\mathrm{Kim} \mathrm{H}-\mathrm{C}$, Jeon UB, et al. Celiac axis and common hepatic artery variations in 5002 patients: systematic analysis with spiral CT and DSA. Radiology. 2010;255(1):278-88. https://doi.org/ 10.1148/radiol.09090389.

2. Hiatt JR, Gabbay J, Busuttil RW. Surgical anatomy of the hepatic arteries in 1000 cases. Ann Surg. 1994;220(1):50-2. PMCid: 1234286.

3. lezzi R, Cotroneo AR, Giancristofaro D, Santoro M, Storto ML. Multidetectorrow CT angiographic imaging of the celiac trunk : anatomy and normal variants. Surg Radiol Anat. 2008;30(4):303-10. DOI: https://doi.org/ 10.1007/s00276-008-0324-7.

4. Sebben GA, Rocha SL, Sebben MA, Parussolo Filho PR, Gonçalves BH. Variations of hepatic artery: anatomical study on cadavers. Rev Col Bras Cir. 2013;40(3):221-6. PMid: 23912370.

5. Duran C, Uraz S, Kantarci M, Ozturk E, Doganay S, Dayangac $\mathrm{M}$, et al. Hepatic arterial mapping by multidetector computed tomographic angiography in living donor liver transplantation. $J$ Comput Assist Tomogr. 2009;33(4):61825. https://doi.org/10.1097/ RCT.0b013e31818d814b.

6. Araujo Neto SA, Franca HA, Mello Junior CF, Silva Neto EJ, Negromonte GRP, Duarte CMA, et al. Anatomical variations of the celiac trunk and hepatic arterial system: an analysis using multidetector computed tomography angiography. Radiol Bras. 2015;48(6):358-62. https://doi.org/ 10.1590/0100-3984.2014.0100.

7. Kuiava VA, Perin AT, Navarini D, Ambros SA, Correa MP. Hepatic arterial pattern and celiac trunk not described in literature. Clin Biomed Res. 2018;38(3):305-7. https://doi.org/ 10.4322/2357-9730.84719.
8. Prakash, Rajini T, Mokhasi V, Geethanjali BS, Sivacharan PV, Shashirekha M. Coeliac trunk and its branches: anatomical variations and clinical implications. Singapore Med J. 2012;53(5):329-31. PMid: 22584973.

9. Koops A, Wojciechowski B, Broering DC, Adam G, Krupski-Berdien G. Anatomic variations of the hepatic arteries in 604 selective celiac and superior mesenteric angiographies. Surg Radiol Anat. 2004;26(3):239-44. https://doi.org/10.1007/s00276-0040229-z.

10. Eubank WB, Wherry KL, Maki JH, Sahin H, Funkhouser CP, Schmiedl UP. Preoperative evaluation of patients awaiting liver transplantation: comparison of multiphasic contrastenhanced 3D magnetic resonance to helical computed tomography examinations. J Magn Reson Imaging. 2002;16(5):565-75. https://doi.org/ 10.1002/jmri.10194.

11. Foley WD, Mallisee TA, Hohenwalter MD, Wilson CR, Quiroz FA, Taylor AJ. Multiphase hepatic CT with a multirow detector $C T$ scanner. AJR Am J Roentgenol. 2000;175(3):679-85. https://doi.org/ 10.2214/ajr.175.3.175067.

12. Khalaf $\mathrm{H}$. Vascular complications after deceased and living donor liver transplantation: a singlecenter experience. Transplant Proc. 2010;42(3):865-70. https://doi.org/10.1016/j. transproceed.2010.02.037.

13. Saylisoy S, Atasoy C, Ersoz S, Karayalçin K, Akyar S. Multislice CT angiography in the evaluation of hepatic vascular anatomy in potential right lobe donors. Diagn Interv Radiol. 2005;11(1):51-9. PMid: 15795845.

14. Redvanly RD, Nelson RC, Stieber AC, Dodd GD III. Imaging in the preoperative evaluation of adult liver transplant candidates: goals, merits of various procedures, and recommendations. AJR Am J Roentgenol. 1995;164(3):611-7. https://doi.org/10.2214/ ajr.164.3.7863881.

15. CoşKun M, Kayahan EM, Özbek O, Çakir B, Dalgiç A, Haberal M. Imaging of hepatic arterial anatomy for depicting vascular variations in living related liver transplant donor candidates with multidetector computed tomography: comparison with conventional angiography. Transplant Proc. 2005;37(2):1070-3. https://doi.org/10.1016/j. transproceed.2005.01.019.

16. Coelho JCU, Matias JEF, Parolin MB, Martins EL, Salvalaggio PRO, Gonçalves CG. Complicações vasculares pós-transplante hepático. Rev Col Bras Cir. 2000;27(6):378-82.

17. Piardi T, Lhuaire M, Bruno O, Memeo R, Pessaux P, Kianmanesh R, Sommacale D. Vascular complications following liver transplantation: a literature review of advances in 2015. World J Hepatol. 2016;8(1):36-57. https://doi.org/10.4254/wjh.v8.i1.36.

18. Paes-Barbosa FC, Ferreira FG, Szutan LA. Hepatectomy preoperative planning. Rev Col Bras Cir. 2010;37(5):370-5. PMid: 21181004.

19. Dazzi FL, Ribeiro-Júnior MAF, Mancero JMP, Gonzalez AM, Leão-Filho HM, Silva AO, D'albuquerque LAC. Os achados de imagem para avaliação da tríade portal são confiáveis para realização do transplante hepático com doador vivo? ABCD Arq Bras Cir Dig. 2013;26(4):296-301. https://doi.org/10.1590/S010267202013000400009 . 